\title{
Signaling message time for homogeneous and heterogeneous protocols: a comparative study
}

\author{
Hadeel Saleh Haj Aliwi ${ }^{1, *}$, Nasser K. A. Alajmi ${ }^{2}$, Putra Sumari ${ }^{1}$, Kamal Alieyan ${ }^{3}$ \\ ${ }^{1}$ School of Computer Sciences, Universiti Sains Malaysia, 11800 Pulau Penang, Malaysia \\ ${ }^{2}$ Saad Al-Abdullah Academy for Security Sciences, Kuwait \\ ${ }^{3}$ National Advanced IPv6 Centre, Universiti Sains Malaysia, 11800 Pulau Penang, Malaysia
}

\section{A R T I C L E I N F O}

\section{Article history:}

Received 2 July 2016

Received in revised form

13 August 2016

Accepted 14 August 2016

Keywords:

Multimedia

Signaling protocols

Translation gateway

Message time

\section{Introduction}

Over the last decade, multimedia techniques have been developed rapidly to enable users to communicate between each other over the Internet using all types of chatting services such as instant messages, audio, and video. However, users cannot phonetically communicate with each other unless they use the same chatting applications since each chatting application has its own control protocol to handle the call setup, the real time media transmission, and the call teardown sessions (Saravanan and Ramadass, 2000).

Due to the appearance of many signaling protocols (Glasmann et al., 2003; Ramadass et al., 1997), media conferencing systems and Internet Protocol (IP) applications, the interoperability has become very necessary to provide full end-to-end connectivity and to give users the flexibility to select their preferred applications as long as there is a method or mechanism that allows bridging the gap between the heterogeneous signaling protocols. Furthermore, the multimedia communication service providers understand that users want to communicate with each other regardless of the service provider and protocol used on their IP

\footnotetext{
* Corresponding Author.

Email Address: Hadeelsaleh12@yahoo.com (H. S. Haj Aliwi)

http://dx.doi.org/10.21833/ijaas.2016.08.003

2313-626X/C) 2016 The Authors. Published by IASE.

This is an open access article under the CC BY-NC-ND license

(http://creativecommons.org/licenses/by-nc-nd/4.0/)
}

network. Thus, the only way to enable the users to communicate using different chatting applications is to design an efficient mapping architecture for any two control protocols used by different chatting applications (Oishi et al., 2007).

This paper provides an analytical comparison between the signaling message time for the homogeneous and heterogeneous protocols environments including the main six heterogeneous mapping architectures.

\section{Homogeneous protocols environment}

In case of two users use the same chatting application which uses the same signaling protocol, so the signaling messages can be exchanges directly between the sender and the receiver as shown in Fig. 1.

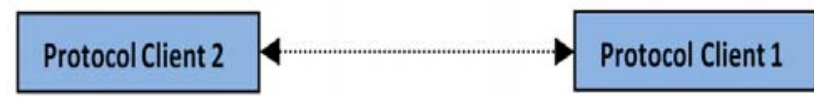

Fig. 1: Homogeneous protocols signaling message exchange

If we consider client 1 message time as $T_{M}(C 1)$, client 2 message time as $T_{M}(C 2)$, sending time by protocol client 1 as $T_{S_{M}}(C 1)$, sending time by protocol client 2 as $T_{S_{M}}(C 2)$, receiving time by protocol client 1 as $T_{R_{M}}(C 1)$, and receiving time by protocol client 2 as $T_{R_{M}}(C 2)$, so

$$
T_{M}(C 1)=T_{R_{M}}(C 2)-T_{S_{M}}(C 1)
$$




$$
T_{M}(C 2)=T_{R_{M}}(C 1)-T_{S_{M}}(C 2)
$$

\section{Heterogeneous protocols environment}

This section presents the variations in the message time of each heterogeneous protocol 1- protocol 2 architecture. Figs. 2, 3, 4, 5, 6, and 7 present the most common six architectures.

Case 1: Both protocol 1 and protocol 2 clients setup and teardown the call directly through the protocol 1-protocol 2 translation gateway (Kolhar, 2010).

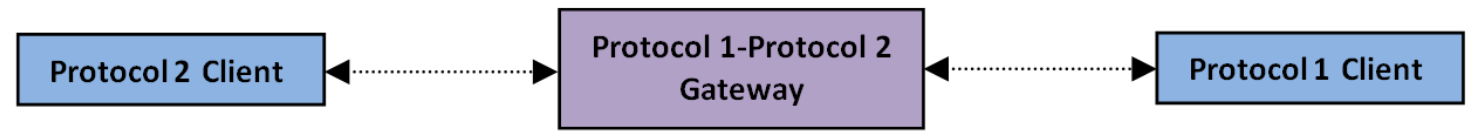

Fig. 2: Heterogeneous protocols signaling message exchange: Case 1

If we consider protocol 1 client Message time as $T_{M}(C 1)$, protocol 2 client message time as $T_{M}(C 2)$, sending time by protocol 1 as $T_{S_{M}}(C 1)$, sending time by protocol 2 as $T_{S_{M}}(C 2)$, receiving time by protocol 1 client as $T_{R_{M}}(C 1)$, receiving time by protocol 2 client as $T_{R_{M}}(C 2)$, sending time by protocol 1protocol 2 gateway as $T_{S_{M}}(G)$, receiving time by protocol 1-protocol 2 gateway as $T_{R_{M}}(G)$, and translation process time inside protocol 1-protocol 2 gateway as $T_{G}$, so

$$
\begin{aligned}
& T_{M}(C 1)=\left(T_{R_{M}}(G)-T_{S_{M}}(C 1)\right)+T_{G}+\left(T_{R_{M}}(C 2)-\right. \\
& \left.T_{S_{M}}(G)\right) \\
& T_{M}(C 2)=\left(T_{R_{M}}(G)-T_{S_{M}}(C 2)\right)+T_{G}+\left(T_{R_{M}}(C 1)-\right. \\
& \left.T_{S_{M}}(G)\right)
\end{aligned}
$$

Case 2: Protocol 1 client deals with the protocol 1-protocol 2 translation gateway with the existence of the client's respective server during the signaling session, whereas, protocol 2's client setup and teardown the call directly through the protocol 1protocol 2 translation gateway (Singh, 2006).

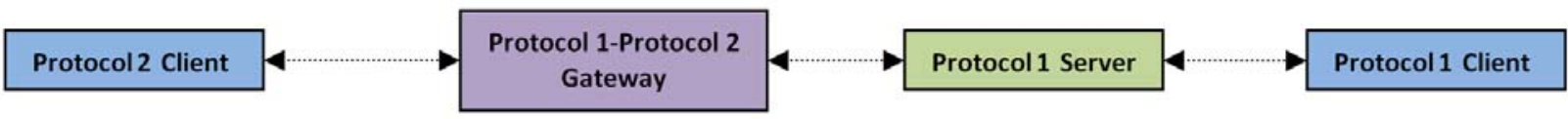

Fig. 3: Heterogeneous protocols signaling message exchange: Case 2

If we consider the sending time by protocol 1 server as $T_{S_{M}}(S 1)$ and receiving time by protocol 1 server as $T_{R_{M}}(S 1)$, and so storage time inside protocol 1 server as $T_{S 1}$

$$
\begin{aligned}
& T_{M}(C 1)=\left(T_{R_{M}}(S 1)-T_{S_{M}}(C 1)\right)+T_{S 1}+\left(T_{R_{M}}(G)-\right. \\
& \left.T_{S_{M}}(S 1)\right)+T_{G}+\left(T_{R_{M}}(C 2)-T_{S_{M}}(G)\right) \\
& T_{M}(C 2)=\left(T_{R_{M}}(G)-T_{S_{M}}(C 2)\right)+T_{G}+\left(T_{R_{M}}(S 1)-\right. \\
& \left.T_{S_{M}}(G)\right)+T_{S 1}+\left(T_{R_{M}}(C 1)-T_{S_{M}}(S 1)\right)
\end{aligned}
$$

Case 3: Protocol 1 client setup and teardown the call directly through the protocol 1-protocol 2 translation gateway, whereas, protocol 2's client deals with the protocol 1-protocol 2 translation gateway with the existence of the client's respective server during the signaling session (Kolhar et al., 2008).

\section{Protocol 2 Client - Protocol 2 Server $-\begin{gathered}\text { Protocol 1-Protocol } 2 \\ \text { Gateway }\end{gathered}$}

Fig. 4: Heterogeneous protocols signaling message exchange: Case 3

If we consider the sending time by protocol 2 server as $T_{S_{M}}(S 2)$ and receiving time by protocol 2 server as $T_{R_{M}}(S 2)$, and so storage time inside protocol 2 server as $T_{S 2}$

$$
\begin{aligned}
& T_{M}(C 1)=\left(T_{R_{M}}(G)-T_{S_{M}}(C 1)\right)+T_{G}+\left(T_{R_{M}}(S 2)-\right. \\
& \left.T_{S_{M}}(G)\right)+T_{S 2}+\left(T_{R_{M}}(C 2)-T_{S_{M}}(S 2)\right)
\end{aligned}
$$

$$
\begin{aligned}
& T_{M}(C 2)=\left(T_{R_{M}}(S 2)-T_{S_{M}}(C 2)\right)+T_{S 2}+\left(T_{R_{M}}(G)-\right. \\
& \left.T_{S_{M}}(S 2)\right)+T_{G}+\left(T_{R_{M}}(C 1)-T_{S_{M}}(G)\right)
\end{aligned}
$$

Case 4: Both protocol 1 and protocol 2 clients deal with the protocol 1-protocol 2 translation gateway with the existence of the client's respective server during the signaling session (Dagiuklas et al., 2005).

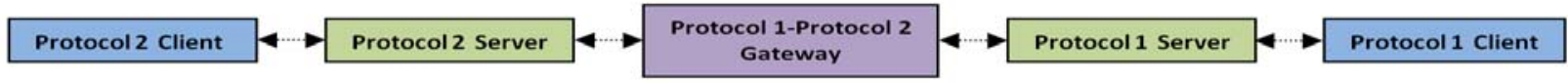

Fig. 5: Heterogeneous protocols signaling message exchange: Case 4

$$
\begin{aligned}
& T_{M}(C 1)=\left(T_{R_{M}}(S 1)-T_{S_{M}}(C 1)\right)+T_{S 1}+\left(T_{R_{M}}(G)-\right. \\
& \left.T_{S_{M}}(S 1)\right)+T_{G}+\left(T_{R_{M}}(S 2)-T_{S_{M}}(G)\right)+T_{S 2}+ \\
& \left(T_{R_{M}}(C 2)-T_{S_{M}}(S 2)\right)
\end{aligned}
$$

$$
\begin{aligned}
& T_{M}(C 2)=\left(T_{R_{M}}(S 2)-T_{S_{M}}(C 2)\right)+T_{S 2}+\left(T_{R_{M}}(G)-\right. \\
& \left.T_{S_{M}}(S 2)\right)+T_{G}+\left(T_{R_{M}}(S 1)-T_{S_{M}}(G)\right)+T_{S 1}+ \\
& \left(T_{R_{M}}(C 1)-T_{S_{M}}(S 1)\right)
\end{aligned}
$$


Case 5: In this architecture, the translation gateway which is responsible for two ways translation matters has been divided into two one way translation gateways; protocol 1-to-protocol 2 translation gateways and protocol 2-to-protocol 1 translation gateway. Both protocol 1 and protocol 2 clients deal with the protocol 1-to-protocol 2 translation gateway and protocol 2-to-protocol 1 translation gateway respectively with the existence of the client's respective server during the signaling session (Saint-Andre et al., 2014).

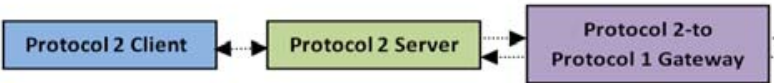

Protocol 1-to

Protocol 2 Gateway

Fig. 6: Heterogeneous protocols signaling message exchange: Case 5

If we consider the sending time by protocol 1-toprotocol 2 gateway as $T_{S_{M}}(G 1)$, receiving time by protocol 1-to-protocol 2 gateway as $T_{R_{M}}(G 1)$, and translation process time inside protocol 1-toprotocol 2 gateway as $T_{G 1}$, sending time by protocol 2-to-protocol 1 gateway as $T_{S_{M}}(G 2)$, receiving time by protocol 2-to-protocol 1 gateway as $T_{R_{M}}(G 2)$, and translation process time inside protocol 2-toprotocol 1 gateway as $T_{G 2}$, so

$$
\begin{aligned}
& T_{M}(C 1)=\left(T_{R_{M}}(S 1)-T_{S_{M}}(C 1)\right)+T_{S 1}+\left(T_{R_{M}}(G 1)-\right. \\
& \left.T_{S_{M}}(S 1)\right)+T_{G 1}+\left(T_{R_{M}}(S 2)-T_{S_{M}}(G 1)\right)+T_{S 2}+ \\
& \left(T_{R_{M}}(C 2)-T_{S_{M}}(S 2)\right)
\end{aligned}
$$

$$
\begin{aligned}
& T_{M}(C 2)=\left(T_{R_{M}}(S 2)-T_{S_{M}}(C 2)\right)+T_{S 2}+\left(T_{R_{M}}(G 2)-\right. \\
& \left.T_{S_{M}}(S 2)\right)+T_{G 2}+\left(T_{R_{M}}(S 1)-T_{S_{M}}(G 2)\right)+T_{S 1}+ \\
& \left(T_{R_{M}}(C 1)-T_{S_{M}}(S 1)\right)
\end{aligned}
$$

Case 6: : Both protocol 1 and protocol 2 clients setup and teardown the call directly through the protocol 1-to-protocol 2 translation gateway and protocol 2-to-protocol 1 translation gateway respectively (Haj Aliwi and Sumari, 2016).

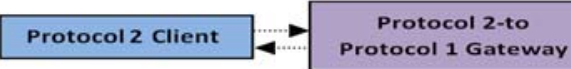

Protocol 1-to Protocol 2 Gateway

Protocol 1 Client

Fig. 7: Heterogeneous protocols signaling message exchange: Case 6

$$
\begin{aligned}
& T_{M}(C 1)=\left(T_{R_{M}}(G 1)-T_{S_{M}}(C 1)\right)+T_{G 1}+\left(T_{R_{M}}(C 2)-\right. \\
& \left.T_{S_{M}}(G 1)\right) \\
& T_{M}(C 2)=\left(T_{R_{M}}(G 2)-T_{S_{M}}(C 2)\right)+T_{G 2}+\left(T_{R_{M}}(C 1)-\right. \\
& \left.T_{S_{M}}(G 2)\right)
\end{aligned}
$$

A flowchart is provided in order to determine the message time of an architecture regardless it is a homogeneous or heterogenous signaling message exchange environment as presented in Fig. 8.

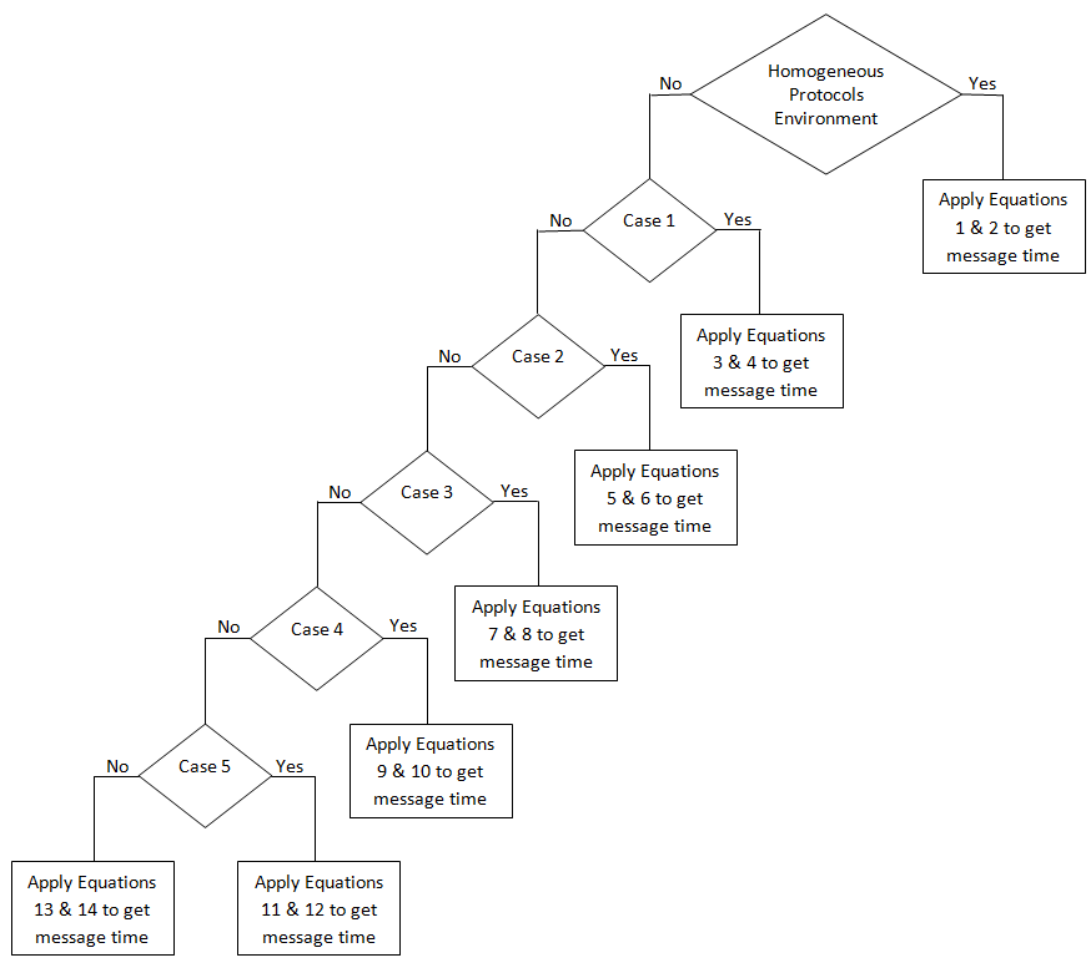

Fig. 8: Message time determination flowchart. 


\section{Experiments and results}

For the experiments, Network Simulation NS2 (ns-2.35) has been used, as well as, two heterogeneous signaling protocols; IAX and Jingle has been chosen and G.711 audio codec has been considered in order to find the message time for each homogeneous and heterogeneous architecture as shown in Table 1. We have tested the IAX-Jingle simulation environment within number of calls varies between 1 and 3 . Based on the obtained results, IAX maintains high call quality with different number of calls due to having trunking property followed by Jingle protocol, case 6 as the best case in the heterogeneous protocols environment, and case 4 as the worst case.

Table 1: Message time for homogeneous and heterogeneous architectures

\begin{tabular}{|c|c|c|c|c|}
\hline \multicolumn{5}{|c|}{ Signaling Message Time (Seconds) } \\
\hline \multicolumn{2}{|c|}{ Number of Calls } & 1 & 2 & 3 \\
\hline \multirow{3}{*}{ Homogeneous } & IAX & 0.002235 & 0.003329 & 0.004008 \\
\cline { 2 - 5 } & Jingle & 0.002344 & 0.003351 & 0.004017 \\
\hline \multirow{4}{*}{ Heterogeneous } & Case 1 & 0.002408 & 0.003383 & 0.004055 \\
\cline { 2 - 5 } & Case 2 & 0.002411 & 0.003395 & 0.004069 \\
\cline { 2 - 5 } & Case 3 & 0.002415 & 0.003391 & 0.004066 \\
\cline { 2 - 5 } & Case 4 & 0.002477 & 0.003431 & 0.004083 \\
\cline { 2 - 5 } & Case 5 & 0.002462 & 0.003417 & 0.004078 \\
\cline { 2 - 5 } & Case 6 & 0.002382 & 0.003378 & 0.004045 \\
\hline
\end{tabular}

\section{Conclusion}

In this paper, we presented the architecture for the homogeneous and the common six heterogeneous signaling protocols environments and calculating the signaling message time for the whole architectures as the message time value varies from architecture to the other one.

\section{References}

Dajiuklas T, Ioannou K and Garmpis AA (2005). A lightweight and scalable VoIP platform based on MGCP/H. 323 interworking and QoS management capabilities. In the $4^{\text {th }}$ WSEAS International Conference on Information Security, Communications and Computers, Tenerife, Spain: 548-553.

Glasmann J, Kellerer W and Muller H (2003). Service architectures in H. 323 and SIP: A comparison. IEEE Communications Surveys and Tutorials, 5(2): 32-47.

Haj Aliwi HS, and Sumari P (2016). An Effective Mapping Architecture between two Heterogeneous Signaling Protocols. Canadian Journal of Pure and Applied Sciences, 10(1): 3755-3762.

Kolhar M (2010). Real-time conference gateway for heterogeneous clients: real-time switching clients and inter-asterisk exchange clients. PhD Thesis, National Advanced IPv6, Universiti Sains Malaysia, Penang, Malaysia.

Kolhar MS, Wan TC, Abouabdalla 0 and Sureswaran R (2008). Multimedia communication: RSW control protocol and IAX. In the International Symposium on High Capacity Optical Networks and Enabling Technologies. IEEE, Penang, Malaysia: 75-79

Oishi T and Inouchi H (2007). Method and system for persistent translation between protocols. US Patent 7305480 [Online]. Accessd July 2015, Available from World Wide Web: http://www.patentstorm.us/patents/pdfs/ patent_id/ 7305480.html

Saint-Andre P, Houri A and Hildebrand J (2014). Interworking between the session initiation protocol (SIP) and the extensible messaging and presence protocol (XMPP): Architecture, addresses, and error Handling. RFC 7247, DOI 10.17487/RFC7247, May 2014, <http://www.rfceditor.org/info/rfc7247>.

Saravanan K and Ramadass S (2000, February). A bidirectional multicast tunneled to support the distributed multimedia conferencing environment architecture. In the International Workshop on Asia Pacific Advanced Network and its Applications. IEEE, Tsukuba, Japan: 135-139.

Singh KN (2006). Reliable scalable and interoperable internet telephony. PhD Thesis, School of Arts and Sciences, Columbia University, Columbia.

Sureswaran R, Subramanian RK, Guyennet H and Trehel M (1997). Using the RSW control criteria to create a distributed environment for multimedia conferencing. In the Research and Development in Computer Science and its Applications, Penang, Malaysia: 27-29. 